\title{
A CHARACTERIZATION OF THE UNIFORM CLOSURE OF THE SET OF HOMEOMORPHISMS OF A COMPACT TOTALLY DISCONNECTED METRIC SPACE INTO ITSELF
}

\author{
FRANK B. MILES
}

\begin{abstract}
The limit index $\lambda(x)$ of a point $x$ in a compact metric space is defined. (Roughly: Isolated points have index 0 , limit points have index 1 , limit points of limit points have index 2 , and so forth.) Then the following theorem is proved.

THEOREM 1. Let $E$ be a compact, totally disconnected metric space. Then the uniform closure of the set of homeomorphisms of $E$ into itself is the set $C_{\lambda}$ of continuous functions $f$ from $E$ to $E$ satisfying

(1) $\lambda(x)<\lambda(f(x))$ for all $x \in E$, and

(2) if $y$ is not a condensation point of $E$, then $f^{-1}(y)$ contains at most one $x$ such that $\lambda(x)=\lambda(y)$.

Further, the set of homeomorphisms of $E$ into $E$ is a dense $G_{\S}$ subset of the complete metric space $C_{\lambda}$.
\end{abstract}

A concept that we will call the limit index of a point in a compact metric space was used by Miles in the proof of a theorem in abstract harmonic analysis [1, Theorem A]. Theorem 1 of this paper can be proved from that theorem. The proof of Theorem 1 presented in this paper is simpler but similar and does not use harmonic analysis. The original form of the category argument used here is due to Kaufman [2]. Adaptations have appeared in [1, 3 and 4].

We first introduce some definitions and notation.

Let $E$ be a compact metric space. For each ordinal $\alpha \leqslant \Omega$ (the first uncountable ordinal), define $E_{\alpha}$ as follows. Let $E_{0}=E$. Let $E_{\alpha+1}$ be the set of limit points of $E_{\alpha}$. If $\beta$ is a limit ordinal, let $E_{\beta}=\bigcap_{\alpha<\beta} E_{\alpha}$. (These definitions are due originally to Cantor [5]. See also Kuratowski [6, p. 261].)

It is shown in [1] and in [6, p. 262] that $E_{\alpha}=E_{\alpha+1}$ for some $\alpha<\Omega$. Let $\alpha_{E}$ be the first ordinal for which this holds and write $\tilde{E}$ for $E_{\alpha_{E}}$. Observe that $\tilde{E}$ is the set of condensation points of $E$.

For a nonempty closed subset $F$ of $E$, define the limit index of $F$, denoted $\lambda(F)$, as follows: If $F \cap \tilde{E} \neq \varnothing$, let $\lambda(F)=\alpha_{E}$; otherwise let $\lambda(F)$ be the last $\alpha$ such that $F \cap E_{\alpha} \neq \varnothing$. (A compactness argument, given in [1], shows that such an $\alpha$ exists.) For $x \in E$, we write $\lambda(x)$ for $\lambda(\{x\})$.

Received by the editors March 10, 1981 and, in revised form, May 23, 1981. The contents of this paper were presented at the University of Illinois Functional Analysis Day on July 3, 1981 and at the Santa Barbara meeting of the American Mathematical Society, November 13-14, 1981.

1980 Mathematics Subject Classification. Primary 54E52; Secondary 54E45, 54B05, 54C35. 
Observe that $\lambda$ has the following properties:

(i) If $\alpha \leqslant \alpha_{E}$, then $\lambda(x) \geqslant \alpha$ if and only if $x \in E_{\alpha}$.

(ii) $\lambda(F)<\alpha_{E}$ implies that $F \cap E_{\lambda(F)}$ is finite.

(iii) $y \in F$ implies that $\lambda(y)<\lambda(F)$.

Let $C(E, E)$ be the set of continuous functions from $E$ to $E$ and $C(E, R)$ be the set of continuous real-valued functions on $E$. Let $C_{\text {fin }}$ be the set of continuous real-valued functions on $E$ with finite range. For $h \in C(E, R)$ and $\varepsilon>0$, let $G(h, \varepsilon)=\left\{f \in C_{\lambda}:\|\gamma \circ f-h\|_{\infty}<\varepsilon\right.$ for some $\left.\gamma \in C_{\text {fin }}\right\}$.

Let $d$ be a metric on $E$ compatible with the topology of $E$. For $f$ and $g$ in $C(E, E)$, let $D(f, g)=\sup \{d(f(x), g(x)): x \in E\}$.

THEOREM 2. Every homeomorphism of $E$ into itself is an element of $C_{\lambda}$.

Proof. Let $f$ be a homeomorphism of $E$ into $E$. The second condition in the definition of $C_{\lambda}$ is trivially satisfied, since $f$ is one-to-one. It remains to show that the first condition holds or, equivalently, that $f\left(E_{\alpha}\right) \subset E_{\alpha}$ for all $\alpha$. Assume that $f\left(E_{\alpha}\right) \subset E_{\alpha}$ is false for some $\alpha$ and let $\beta$ be the first ordinal for which this happens. We will show that this leads to a contradiction. We have $f\left(E_{\beta}\right) \not E_{\beta}$, but, for $\alpha<\beta, f\left(E_{\alpha}\right) \subset E_{\alpha}$. Thus, there is an $x \in E_{\beta}$ such that $y=f(x) \notin E_{\beta}$. Let $\lambda(y)=$ $\alpha$. Then $\alpha<\beta$. Consider $g=\left.f\right|_{E_{\alpha}}$. Clearly, $g$ is a homeomorphism of $E_{\alpha}$ into $E_{\alpha}$. Since $y$ is an isolated point of $E_{\alpha}, g^{-1}(y)=x$ is an isolated point of $E_{\alpha}$. But $x \in E_{\beta}$ and is therefore a limit point of $E_{\alpha}$, so we have a contradiction.

THEOREM 3. $C_{\lambda}$ is complete in the topology of uniform convergence.

Proof. See [1].

LEMMA 1. Let $x_{1}, \ldots, x_{n}$ be distinct elements of $E$; let $g \in C_{\lambda}$ and let $\eta>0$. Then there are distinct elements $y_{1}, \ldots, y_{n}$ of $E$ such that $\lambda\left(x_{j}\right) \leqslant \lambda\left(y_{j}\right)$ and $d\left(y_{j}, g\left(x_{j}\right)\right)<$ $\eta$ for $1 \leqslant j \leqslant n$.

Proof. See [1].

LEMMA 2. Each $G(h, \varepsilon)$ is dense in $C_{\lambda}$.

Proof. Fix $h \in C(E, R)$ and $\varepsilon>0$. Let $g \in C_{\lambda}$ and $\eta>0$. We will show that there is an $f \in G(h, \varepsilon)$ such that $D(f, g)<\eta$.

Write $E=\cup_{j=1}^{n} F_{j}$, where the $F_{j}$ are pairwise disjoint, nonvoid, open and closed subsets of $E$, and where $h$ varies less than $\varepsilon$ and $g$ varies less than $\eta / 2$ on each $F_{j}$. Let $\lambda\left(F_{j}\right)=\alpha_{j}$. If $\alpha_{j}<\alpha_{E}$, then $F_{j} \cap E_{\alpha_{j}}$ is finite, so that we may suppose without loss of generality that $F_{j} \cap E_{\alpha_{j}}$ consists of a single point $x_{j}$. If $\alpha_{j}=\alpha_{E}$, let $x_{j}$ be any point of $F_{j} \cap E_{\alpha_{j}}$. By Lemma 1 , there are distinct $y_{1}, \ldots, y_{n}$ such that $\lambda\left(y_{j}\right) \geqslant \lambda\left(x_{j}\right)$ and $d\left(y_{j}, g\left(x_{j}\right)\right)<\eta / 2,1 \leqslant j \leqslant n$. Define $f(x)=y_{j}$ when $x \in F_{j}$. Then $f \in C_{\lambda}$ and $D(f, g)<\eta$. Now write $E=\cup_{j=1}^{n} A_{j}$, where the $A_{j}$ are disjoint open and closed sets and $y_{j} \in A_{j}, 1 \leqslant j \leqslant n$. Define $\gamma \in C_{\text {fin }}$ by $\gamma(y)=h\left(x_{j}\right)$ when $y \in A_{j}$. Then, when $x \in F_{j}$, we have $|\gamma \circ f(x)-h(x)|=\left|h\left(x_{j}\right)-h(x)\right|<\varepsilon$, so $\|\gamma \circ f-h\|_{\infty}<\varepsilon$.

LeMMA 3. Each $G(h, \varepsilon)$ is open in $C_{\lambda}$. 
Proof. Fix $h \in C(E, R)$ and $\varepsilon>0$. Let $g \in G(h, \varepsilon)$ and let $\gamma \in C_{\text {fin }}$ be such that $\|\gamma \circ g-h\|_{\infty}<\varepsilon$. Let the range of $\gamma$ be $\left\{y_{1}, \ldots, y_{n}\right\}$ and let $F_{j}=\gamma^{-1}\left(y_{j}\right)$, $1 \leqslant j \leqslant n$. Let $\eta>0$ be such that $\eta<\min _{i \neq j}\left\{\operatorname{dist}\left(F_{i}, F_{j}\right)\right\}$. Then if $f \in C_{\lambda}$ and $D(f, g)<\eta$ we have for all $x$ that $f(x) \in F_{j}$ if and only if $g(x) \in F_{j}$, and, hence, $\gamma \circ f=\gamma \circ g$, so $\|\gamma \circ f-h\|_{\infty}<\varepsilon$.

Proof of Theorem 1. Let $f \in C_{\lambda}$. Then $f$ is a homeomorphism of $E$ into $E$ if and only if $f$ is one-to-one. Also, if $f$ is not one-to-one, it is clear that there are an $h \in C(E, R)$ and $\varepsilon>0$ such that $f \notin G(h, \varepsilon)$. It follows that $f$ is a homeomorphism of $E$ into $E$ if and only if $f$ is in every $G(h, \varepsilon)$.

Let $\left\{h_{n}\right\}_{n=1}^{\infty}$ be dense in $C(E, R)$. Then $f$ is a homeomorphism of $E$ into $E$ if and only if $f$ is in $\bigcap_{n, k=1}^{\infty} G\left(h_{n}, k^{-1}\right)$. Combining this with Theorem 3 and Lemmas 2 and 3 and applying the Baire Category Theorem, we see that the homeomorphisms in $C_{\lambda}$ form a dense $G_{\delta}$ subset of the complete metric space $C_{\lambda}$. This, together with Theorem 2, completes the proof.

\section{REFERENCES}

1. F. B. Miles, Compact, totally disconnected sets that contain K-sets, Michigan Math. J. 21 (1974), 315-319.

2. R. Kaufman, A functional method for linear sets, Israel J. Math. 5 (1967), 185-187.

3. Y. Katznelson, An introduction to harmonic analysis, Wiley, New York, 1968, pp. 184-185.

4. F. B. Miles, Existence of special $K$-sets in certain locally compact abelian groups, Pacific J. Math. 44 (1973), 219-232.

5. G. Cantor, Ueber enendliche, lineare Punktmannichfaltigkeiten, Math. Ann. 17 (1880), 355-358.

6. K. Kuratowski, Topology, Vol. I, Academic Press, New York, 1966.

Department of Mathematics, California State University, Dominguez Hills, Carson, CaliFORNIA 90747 